\section{(2) OPEN ACCESS}

\title{
Should antidepressants be used for major depressive disorder?
}

\section{Janus Christian Jakobsen $\odot{ }^{1,2,3}$ Christian Gluud, ${ }^{1}$ Irving Kirsch ${ }^{4}$}

\subsection{6/bmjebm-2019-111238}

${ }^{1}$ The Copenhagen Trial Unit, Centre for Clinical Intervention Research, Rigshospitalet, Copenhagen University Hospital, Copenhagen, Denmark

${ }^{2}$ Department of Cardiology, Holbaek Hospital, Holbaek, Denmark

${ }^{3}$ Department of Regional Health Research, The Faculty of Heath Sciences, University of Southern Denmark, Denmark

${ }^{4}$ Program in Placebo Studies, Harvard Medical School, Boston, Massachusetts, USA

\section{Correspondence to:} Dr Janus Christian Jakobsen, The Copenhagen Trial Unit, Centre for Clinical Intervention Research, Copenhagen 2100, Denmark; janusjakobsen@ mac.com

\section{Abstract}

Background Major depressive disorder is estimated by the WHO to affect more than 300 million people globally, making depression the leading cause of disability worldwide. Antidepressants are commonly used to treat depression.

Objective The study aimed to provide an update on the evidence on the effects of antidepressants compared with placebo. Should antidepressants be used for adults with major depressive disorder?

Study selection We searched the Cochrane Library, BMJ Best Practice and PubMed up to June 2019 with the search terms 'depression' and 'antidepressants' targeting reviews published in English since 1990.

Findings Several reviews have assessed the effects of antidepressants compared with placebo for depression. Generally, all the previous reviews show that antidepressants seem to have statistically significant effects on depressive symptoms, but the size of the effect has questionable importance to most patients. Antidepressants seem to have minimal beneficial effects on depressive symptoms and increase the risk of both serious and nonserious adverse events.

Conclusions The benefits of antidepressants seem to be minimal and possibly without any importance to the average patient with major depressive disorder. Antidepressants should not be used for adults with major depressive disorder before valid evidence has shown that the potential beneficial effects outweigh the harmful effects.

\section{Introduction}

Major depressive disorder is estimated by the WHO to affect more than 300 million people globally, making the disorder the leading cause of disability worldwide. ${ }^{1}$ The lifetime prevalence of major depressive disorder is between 10\% and $20 \% .^{2}{ }^{3}$ Antidepressants are commonly used to treat depression. We provide a narrative review on the evidence on the effects of antidepressants compared with placebo in patients with a primary diagnosis of major depressive disorder. We do not consider the effects of antidepressants in patients with a primary somatic disorder or the effects of antidepressants for other disorders than major depressive disorder.

\section{How is major depressive disorder diagnosed?}

The diagnosis of major depressive disorder in clinical practice is based on the presence of certain symptoms according to the diagnostic criteria outlined in either the Diagnostic and Statistical Manual of Mental Disorders, Fifth Edition (DSM-5) or the International Classification of Diseases-10th Revision. ${ }^{45}$ These two sets of diagnostic criteria are largely comparable. The diagnosis of depression as it is defined by these diagnostic criteria does not build on any aetiological or pathophysiological framework. The assessment according to the diagnostic criteria might be supported by a structured interview, such as the Mini-International Neuropsychiatric Interview or the Structured Clinical Interview for DSM. ${ }^{67}$ The conduct of these interviews may take 15-60 min. There are no objective tests and no laboratory test (eg, blood tests, MRI scans) for depression or for validating the diagnosis.

\section{Antidepressants}

Antidepressant use is considerable, especially in the Western world, and is on the rise in several countries. $^{8}$ Data from the National Health and Nutrition Examination Survey published in 2017 showed that during 2011-2014 about one in eight people aged 12 and over in the USA reported taking antidepressants during the previous month. ${ }^{9}$ Antidepressant use increased nearly 65\% over a 15 -year time frame, ${ }^{9}$ and more than $60 \%$
Check for updates

( $)$ Author(s) (or their employer(s)) 2020. Re-use permitted under CC BY-NC. No commercial re-use. See rights and permissions. Published by BMJ.

To cite: Jakobsen JC, Gluud C, Kirsch I. BMJ Evidence-Based Medicine 2020;25:130-136.

\section{Sources and selection criteria}

Two investigators (JCJ and CG) independently searched the Cochrane Library, BMJ Best Practice and PubMed up to June 2019 with the search terms 'depression' and 'antidepressants' targeting narrative and systematic reviews published in English since 1990. We included any review assessing benefits and harms of any antidepressant compared with placebo in adults. We additionally searched references of the identified articles and, where relevant, we included recommendations from guidelines. We have not published a protocol before performing the literature search, which is a limitation. 


\section{Box 1 Types of antidepressants}

Selective serotonin reuptake inhibitors (eg, citalopram, escitalopram, fluoxetine, paroxetine and sertraline).

Serotonin-norepinephrine reuptake inhibitors (eg, venlafaxine, duloxetine and milnacipran).

- Tricyclic antidepressants (eg, amitriptyline, imipramine and nortriptyline).

- Atypical antidepressants (eg, mirtazapine, agomelatine and bupropion).

- Monoamine oxidase inhibitors (eg, isocarboxazid and phenelzine).

of people in the USA taking antidepressants have been taking them for more than 2 years. ${ }^{9}$ Antidepressants for major depressive disorder, either alone or in combination with psychotherapy, are recommended by the UK National Institute for Health and Care Excellence (NICE), the American Psychiatric Association, as well as guidelines. ${ }^{10-16}$ Furthermore, psychiatrists and general practitioners worldwide routinely use antidepressants in the treatment of depression.

Several different antidepressants exist (see box 1). ${ }^{17}$ Selective serotonin reuptake inhibitors (SSRIs) are the most widely prescribed class of antidepressants and are often first-line treatment for depression. ${ }^{18}$

\section{How much difference should antidepressants make?}

The effect of antidepressants on depression is, in clinical trials, most often measured by assessing the effect on the severity of depressive symptoms on a scale, such as the 17-item Hamilton Depression Rating Scale (HDRS) (range 0-52 points). The HDRS is the most commonly used depression rating scale and is the recommended scale by psychiatrists worldwide. NICE previously suggested that a three-point difference on the HDRS or a 0.5 standardised mean difference (SMD) met the criteria for clinical significance (minimally important difference). ${ }^{19}$ NICE no longer presents these thresholds for clinical significance on their website and these thresholds have been criticised. Nevertheless, these thresholds have since been used in several studies assessing the effects of antidepressants. ${ }^{2021}$ Furthermore, the 0.5 SMD threshold was originally proposed by Cohen (as a minimum for a 'moderate' effect) and has been used as a minimally important difference in several studies across medical specialties. ${ }^{22}$

The above-mentioned thresholds suggested by NICE were not empirically based and are presumably too small. ${ }^{23}$ One study has shown that an SSRI-placebo mean difference of up to three points on the HDRS corresponds to a clinical judgement of 'no change' in the person's condition. ${ }^{23}$ Based on these data, a more stringent criterion of clinical significance has been recommended. ${ }^{24}$ Thus, global clinical ratings of 'minimal improvement' correspond to approximately a seven-point change on the $\mathrm{HDRS}^{23}$ or an SMD of 0.875 . These have been proposed as empirically derived thresholds for a minimally important difference. ${ }^{24}$ It should, however, be noted that these empirically derived thresholds do not necessarily provide a precise estimate of what patients consider the smallest worthwhile effect of antidepressants. Nevertheless, these suggested thresholds for clinical significance need to be considered when interpreting the results of reviews on depression; as mentioned all available evidence shows that a difference of, for example, two HDRS points must be regarded as a minimal effect and is presumably undetectable by the average patient with depression. ${ }^{23}$ However, although the available evidence (based on the available assessment scales) shows that the average effect on depressive symptoms is minimal, theoretically and in clinical practice some patients might benefit significantly from antidepressants. Nevertheless, if the averaged effect is minimal and close to zero effect and some patients benefit significantly from antidepressants, then there has to be a comparable proportion of patients who are significantly harmed by antidepressants-otherwise the averaged effect would not be close to zero effect. Moreover, we have not identified any studies that identify clearly who will respond to antidepressants and who will not.

Trialists often dichotomise the HDRS scale, that is, transforming the HDRS score between 0 and 52 into a binary score, for example when responders are compared with non-responders based on a criterion of $\geq 50 \%$ improvement on the HDRS (yes/ no). However, several publications have shown that such transformation of continuous data into two groups (dichotomisation) is problematic due to several methodological limitations of dichotomisation, and dichotomisation has been shown to produce biased results. ${ }^{25}$ Furthermore, a person who improves by $\geq 50 \%$ is called a responder, whereas one who improves by $49 \%$ is called a nonresponder, thus inflating the apparent difference between these patients. ${ }^{26}$ Conversely, a person who improves by $\geq 50 \%$ is categorised as being the same as a person whose symptoms disappear completely, and one with 49\% improvement is considered the same as a person showing no improvement at all. It will depend on how data are distributed, but even though a larger proportion of participants cross the arbitrary cut-point in the antidepressant group (which has a slightly lower mean HDRS score than the control group) compared with the control group (often HDRS below 8 for remission and 50\% HDRS reduction for response), the effect measured on the HDRS might still be limited to a few HDRS points (eg, 3 HDRS points) or less. If data are distributed differently or other cut-off points are chosen, a 'true' difference between two groups might not be detected when assessing these dichotomised outcomes. Hence, when assessing such dichotomised outcomes, there is a considerable risk of overestimating benefit, but there is also a risk of not detecting a 'true' effect. Hence, dichotomised outcome results, such as 'response' or 'remission', should not be used to assess statistical or clinical significance and should be interpreted with caution.

The HDRS has been questioned as an 'interval scale', that is, that the distance between any two consecutive points is the same wherever you are on the scale. ${ }^{27}$ It must also be noted that when other assessment scales are used (eg, Beck Depression Inventory, Montgomery-Åsberg Depression Rating Scale, Hamilton six-item scale), the results correspond to the HDRS results, that is, statistically significant results with questionable importance to the average patient. ${ }^{28}$ It is possible that the HDRS should be considered ordinal scaled data where the distance between any two consecutive points cannot be said to be the same as the difference between two consecutive points elsewhere on the scale. ${ }^{27}$ It has been argued that the HDRS is psychometrically and conceptually flawed. ${ }^{29}$ Consequently, it may be argued that it is not even possible to assess the clinical relevance of a given HDRS score, and that the evidence base is thus fundamentally flawed due to the reliance on the HDRS. New trials using more clinically relevant outcome scales, in addition to better designs, would 
be needed to demonstrate that antidepressants offer more benefit than harm before antidepressants should be used in the treatment of depression.

In summary, it is of utmost importance to consider the clinical relevance of statistically significant results when assessing the effects of antidepressants and to assess effect sizes based on non-dichotomised scores.

\section{How good are antidepressants?}

Several reviews have assessed the effects of antidepressants compared with placebo for depression. ${ }^{30}$ All these previous reviews show that antidepressants have statistically significant effects on depressive symptoms. The vast majority of the previous reviews were non-systematic reviews (according to the Preferred Reporting Items for Systematic Reviews and Meta-Analyses (PRISMA)), that is, narrative reviews. ${ }^{31}$ We will in the following paragraphs describe two of the largest and recently published systematic reviews. ${ }^{31}$

In 2017 we published a systematic review (according to PRISMA) where all relevant databases were searched, both beneficial and harmful effects were systematically assessed, and a predefined assessment of the clinical significance of antidepressants was performed. ${ }^{28}$ That review found, as all other previous reviews have shown, that SSRIs compared with placebo had a statistically significant effect on depressive symptoms. ${ }^{28}$ The effect size of SSRIs (1.94 HDRS points, 95\% CI -2.50 to -1.37 ; or -0.23 SMD, 95\% CI -0.31 to -0.14 ) was, however, below the predefined threshold for clinical significance (the above-mentioned NICE criteria) and far from 'minimal improvement' (eg, seven-point change on the HDRS or an SMD of 0.875). The few trials assessing long-term effects showed that such effects seemed even smaller than the short-term effects. There were almost no data on suicidal behaviour or quality of life. We found that SSRIs significantly increased the risk of both serious and non-serious adverse events. All the included trials in the review were at high risk of bias, so there is a great risk that the results of the review overestimate the beneficial effects and underestimate the harmful effects of SSRIs. ${ }^{32-34}$ We concluded that the harmful effects of SSRIs seem to outweigh the possible small beneficial effects. Our Grading of Recommendations Assessment, Development and Evaluation (GRADE) assessment of the certainty of the evidence: very low certainty (http://www.gradeworkinggroup.org/).

A large network meta-analysis was recently published in The Lancet. ${ }^{35}$ The authors included placebo-controlled and head-tohead trials of 21 commonly used antidepressants, including SSRIsBox 2. The authors recorded all outcomes as close to 8 weeks as possible, that is, only short-term results were assessed. The authors also assessed 'acceptability' (treatment discontinuation measured by the proportion of patients who withdrew for any reason) and the proportion of patients who dropped out early because of adverse events, but these outcomes are difficult to interpret clinically-patients might, for example, continue taking antidepressants even if they experience serious adverse effects. Neither serious nor non-serious adverse events were assessed in the network meta-analysis. The results regarding benefit were virtually identical with previous reviews and showed that overall antidepressants compared with placebo seemed to reduce depressive symptoms with a statistically significant effect (SMD 0.30, 95\% credibility interval 0.26 to 0.34 ). Such an effect size is presumably without any clinical relevance. ${ }^{24}$ Baseline depression severity was high (HDRS 25.7). A major limitation of the review was that only $18 \%$ of the included trials were assessed at low risk of bias, so there is a great risk that the results of the review overestimate the beneficial effects and underestimate the harmful effects of antidepressants. ${ }^{32-34}$ Our GRADE assessment of the certainty of the evidence: very low certainty.

As mentioned, several other reviews have been published generally confirming that antidepressants have statistically significant effects, but only a few of these reviews assessed the clinical significance of the review results. Nevertheless, if the effect estimates from the previous reviews are related to, for example, the NICE criteria (see 'How much difference should antidepressants make?' section), then the previous reviews confirm that antidepressants generally have minimal beneficial effects on depressive symptoms. Even though there is no valid evidence showing beneficial effects of antidepressants for depression, we cannot exclude that the evidence is showing beneficial effects for other conditions than major depressive disorder.

\section{Limitations of current evidence for antidepressants}

The primary limitation of all the available evidence on the effects of antidepressants is that the majority of previous trials assessing the effects of antidepressants have been at high or unclear risk of bias. ${ }^{28}{ }^{33-35}$ Even if the trials used matching placebo, due to easily recognised adverse events in the experimental group and lack of adverse effect in the control groups, some patients might be able to figure out if they received an antidepressant or a placebo, which might compromise blinding and hence valid assessment of subjective symptoms. Trials at high or unclear risk of bias tend to overestimate benefits and underestimate harms..$^{302-3436-41}$ Despite biases inflating beneficial effects of review results, they still show only negligible differences between antidepressants and placebo on depressive symptoms, and the 'true' effect of antidepressants might not even be statistically significant.

The evidence base for antidepressants is also limited by low generalisability due to inclusion of highly selected patients. Results from a large trial conducted in a clinical setting (the Sequenced Treatment Alternatives to Relieve Depression (STAR*D) trial) showed that $77.8 \%$ of the patients in the STAR*D trial would have been excluded in conventional clinical trials. ${ }^{42}$ The patients in this trial showed a low improvement on the HDRS of 6.6 points after 3 months of treatment with an SSRI, compared with a mean improvement of 14.8 points on the HDRS in conventional comparator trials that, like the STAR*D trial, do not include placebo controls. ${ }^{43}$ These data indicate that, in a clinical setting, the benefit of antidepressants is low and that the exclusion criteria typically used in randomised clinical trials lead to inflated effect estimates.

\section{Industry involvement in research and the possible implications}

Lundh et $a l^{39}$ showed that industry involvement by the manufacturing company leads to more favourable results and conclusions than sponsorship by other sources. Industry-sponsored studies showed more often benefits, relative risk (RR) 1.27 (95\% CI 1.17 to 1.37 ), and more often favourable conclusions, RR 1.34 (95\% CI 1.19 to 1.51$).^{39}$

Ebrahim et $a l^{30}$ identified 185 eligible meta-analyses assessing the effects of different antidepressants, and in the meta-analyses including an author who was an employee of the manufacturer of the assessed drug, these were 22-fold less likely to have negative statements about the drug than other meta-analyses.

The above-mentioned systematic review assessing the effects of SSRIs (see 'How good are antidepressants?' section) also concluded that the vast majority of the included trials (39 out of 
43 trials) with useful data were at high risk of 'for profit' bias. ${ }^{28}$ That review found no significant effect of SSRIs on the HDRS in trials at low risk of 'for profit' bias, whereas the beneficial effects were statistically significant in trials at high or unclear risk of 'for profit' bias. $^{28}$

As a substantial proportion of research concerning antidepressants is prone to 'for profit bias', the results of previous results likely overestimate benefit and underestimate harm. ${ }^{30} 39$ This needs to be considered when interpreting the available research results.

\section{Whom to treat?}

One small individual patient data meta-analysis found support for increasing benefit with increasing severity of depression, ${ }^{20}$ but three larger individual patient data meta-analyses did not find any such effect of antidepressants compared with placebo on depression severity.${ }^{44-46}$ Other studies have found support for increasing benefit with increasing severity of depression, ${ }^{21}$ but the effects size even for patients with the most severe depression was still minimal. ${ }^{21}$ Thus, it appears that there is no clear evidence to support the notion that antidepressants would be of more benefit in severe depression compared with mild or moderate depression.

\section{How long to treat?}

Most trials and reviews have only assessed short-term effects (approximately 4-8weeks) of antidepressants. The long-term effects of antidepressants are unclear. There are very few data on the long-term effects of antidepressants (eg, after 1 year). A recently published review assessing results after 24 weeks showed that the longer-term effects of antidepressants (SMD 0.34) seem as small as the short-term effects. ${ }^{47}$ A clinical practice guideline from NICE showed similar results (SMD 0.28). ${ }^{19}$ It is possible that long-term treatment with antidepressants may even worsen outcomes. ${ }^{48}$ Given the absence of evidence for benefits, there is thus no supporting evidence for long-term treatment with antidepressants.

\section{What are the harms?}

It has been shown that SSRIs, the most commonly used antidepressants, increase the risk of both serious and non-serious adverse events. ${ }^{28}$ Even though the increased relative risk of a serious adverse event is quite large, the absolute risk of a serious adverse event is still small, while SSRI adverse events not considered severe are more common. During long-term use of SSRIs, the most troubling adverse effects seem to be gastrointestinal problems, sleep disturbances and sexual dysfunction, ${ }^{49}$ of which the latter, in some patients, may be persistent even after stopping treatment. ${ }^{50}$ Additionally, the risk of birth defects seems to be increased in infants of women treated with some SSRIs during pregnancy. $^{51}$

Adverse effects of other types of antidepressant than SSRIs (eg, serotonin-norepinephrine reuptake inhibitors and tricyclic antidepressants (TCAs)) are not thoroughly assessed in systematic reviews, but they may, theoretically, be more severe. Nonrandomised studies have, for example, shown that TCAs can cause seizures as well as death due to the slowing of intraventricular conduction, leading to complete heart block or ventricular arrhythmias. ${ }^{49}$ Moreover, because the evidence base consists of short-term trials, it is likely that the current estimates of adverse effects of antidepressants are underestimated. ${ }^{49}$ The focus on short-term follow-up results is problematic in general as a large proportion of patients report taking antidepressants for years. ${ }^{9}$

\section{Withdrawal symptoms}

Withdrawal symptoms after SSRIs typically occur within a few days from drug discontinuation and last a few weeks, also with gradual tapering, but the withdrawal symptoms might include late onset and longer persistence of disturbances and may be easily misidentified as signs of impending relapse. ${ }^{52}$ There are many similarities between the withdrawal symptoms associated with SSRIs and the withdrawal symptoms associated with antidepressants such as venlafaxine and duloxetine. ${ }^{52} \mathrm{~A}$ recently published review found that a significant proportion of people who experience withdrawal symptoms after treatment with antidepressants do so for more than 2 weeks and that it is not uncommon for people to experience withdrawal for several months. ${ }^{53}$ Withdrawal symptoms may be alleviated by reinstating treatment with the antidepressant that triggered the symptoms, and it may for this, and many other reasons, be difficult for some patients to stop taking antidepressants once the treatment has started. ${ }^{52}$ Withdrawal symptoms might also explain why some studies have alleged to show that the risk of relapse seems to be reduced if antidepressants are continued instead of not continued. ${ }^{545}$ Withdrawal symptoms might be the reason why patients who do not continue antidepressants might do worse compared with patients who continue antidepressants.

\section{Combination of antidepressants and psychotherapy}

Antidepressants in combination with psychotherapy are recommended for major depressive disorder by both NICE and the American Psychiatric Association, as well as other guidelines. 51314165657 A non-systematic review showed that adding antidepressants to psychotherapy seemed to have a statistically significant effect, but again the effect was minimal and below the NICE criteria (SMD 0.35; 95\% CI 0.24 to $0.45 ; \mathrm{p}<0.001$ ). ${ }^{10}$ At long-term follow-up, no difference between psychological and combined treatments was found. ${ }^{10}$ Two other reviews have shown similar results. ${ }^{58}{ }^{59}$ The effects of adding antidepressants to psychotherapy seem negligible just as the effects of antidepressants when used as the only therapy.

\section{Other treatment options?}

For persons with depression, a range of options other than antidepressants could be considered when informing patients about treatment choices. Such options involve different forms of psychotherapy, addressing social and psychological issues, physical health, and lifestyle. Choosing between treatment options should be based on careful information about the benefits and harms of each treatment option and alternative treatment options. International guidelines, as a first step, recommend sleep hygiene institution and implementing lifestyle changes, such as adopting regular exercise, ${ }^{14} 16$ smoking cessation and a healthy diet, ${ }^{14} 16$ tapering of drugs that can lower mood, and addressing substance use if relevant ${ }^{16}$-although it must be noted that the evidence behind these suggested interventions is lacking. ${ }^{60}$ Nevertheless, addressing and acknowledging social causes of depression should in all cases be part of the management of depression. Unemployment ${ }^{61}$ and increase in material hardship, such as financial strain, deprivation and poverty, ${ }^{62}$ which are interrelated, increase the risk of depression. Thus, unemployment increases the risk of experiencing conditions that contribute to and perpetuate psychological distress, such as relative poverty, financial stress, loss of personal control and autonomy, and poor social support. ${ }^{61}$ While such determinants 
of depression should effectively be addressed on a societal level, their recognition may for the individual be important in the process of seeking ways to alleviate depression. Importantly, evidence indicates that a reduction in depressive symptoms may not be the single most important outcome to patients, but rather factors such as the ability to participate in everyday activities and return to work. ${ }^{6364}$

\section{What type of future research is needed?}

Based on current evidence, antidepressants seem to offer more harms than benefits. Large randomised clinical trials at low risk of bias, including the use of 'active placebo' (a matching placebo that produces noticeable adverse effects that may convince the person being treated that they are receiving an active intervention) should be conducted. In addition to assessing depressive symptoms and quality of life, harmful effects should be systematically assessed, including long-term follow-up. Such trials should be powered to confirm or reject that antidepressants increase the risk of suicides, hospitalisation, risk of death and so on (the exact sample size will depend on the incidence of events in the control group).

Correction notice This article has been corrected since it was published Online First. The competing interest section now reads "Irving Kirsch is the author of "The Emperor's New Drugs" for which he currently receives occasional royalties."

Contributors JCJ wrote the first draft. All authors approved the final version, and JCJ is the guarantor.

Funding The Copenhagen Trial Unit supported this project with part-time salary for JCJ and CG during the writing of the manuscript.

\section{How patients were involved in the creation of this} article?

We invited a patient who had previously suffered from depression and received treatment as both an inpatient and in an outpatient clinical as well as in primary care to contribute to the article. The patient has been taking antidepressants for 2 years.

- The patient representative specified that it should not be expected that patients with depression have the skills to interpret the validity of research results. Hence, if any treatment for depression should be implemented, then unambiguous valid evidence should have shown that this intervention offers more benefits than harms.

- The patient representative was familiar with the Hamilton Depression Rating Scale (HDRS) and would consider a small improvement (not otherwise specified) on the HDRS of relevance compared with an alternative treatment provided the harms associated with the treatment did not outweigh the benefit.

- After discussing the article with the patient representative, we included more information about the potential adverse effects of antidepressants (see 'What are the harms?' section) and balancing benefits and harms.
Competing interests Irving Kirsch is the author of "The Emperor's New Drugs" for which he currently receives occasional royalties.

Patient consent for publication Not required.

Provenance and peer review Not commissioned; externally peer reviewed.

Data availability statement All data relevant to the study are included in the article.

Open access This is an open access article distributed in accordance with the Creative Commons Attribution Non Commercial (CC BY-NC 4.0) license, which permits others to distribute, remix, adapt, build upon this work non-commercially, and license their derivative works on different terms, provided the original work is properly cited, appropriate credit is given, any changes made indicated, and the use is non-commercial. See: http://creativecommons.org/licenses/by-nc/4.0/.

\section{ORCID iD}

Janus Christian Jakobsen http://orcid.org/0000-0002-3642-2120

\section{References}

1 World Health Organization (WHO). Depression (fact sheet), 2018. Available: http://wwwwhoint/mediacentre/factsheets/fs369/en/ [Accessed Apr 2018].

2 Lim GY, Tam WW, Lu Y, et al. Prevalence of depression in the community from 30 countries between 1994 and 2014. Sci Rep 2018;8:2861-61.

3 Hasin DS, Sarvet AL, Meyers JL, et al. Epidemiology of Adult DSM-5 Major Depressive Disorder and Its Specifiers in the United States. JAMA Psychiatry 2018;75:336-46.

4 WHO. The ICD-10 classification of mental and behavioral disorders: clinical descriptions and diagnostic guidelines. Available: http://www.who. int/classifications/icd/en/bluebook.pdf [Accessed Mar 2018].

5 American Psychiatric Association. Practice guideline for the treatment of patients with major depressive disorder. 3rd edn, 2010.

6 First M, Spitzer R, Gibbon M, et al. Structured clinical interview for DSMIV axis disorders. Washington, DC: American Psychiatric Association, 1997.

7 Sheehan DV, Lecrubier Y, Sheehan KH, et al. The Mini-International neuropsychiatric interview (M.I.N.I.): the development and validation of a structured diagnostic psychiatric interview for DSM-IV and ICD-10. J Clin Psychiatry 1998;59(Suppl 20):22-33. quiz 34-57.

8 OECD. "Antidepressant drugs consumption, 2000 and 2015 (or nearest year)”, in Pharmaceutical sector, OECD Publishing, Paris, 2017. Available: http://dx.doi.org/10.1787/health_glance-2017-graph181-en

9 Pratt LA, Brody DJ, Gu Q. Antidepressant use among persons aged 12 and over: United States, 2011-2014. NCHS data brief, no 283. Hyattsville, MD: National Center for Health Statistics, 2017.

10 Cuijpers P, van Straten A, Warmerdam L, et al. Psychotherapy versus the combination of psychotherapy and pharmacotherapy in the treatment of depression: a meta-analysis. Depress Anxiety 2009;26:279-88.

11 Barbateskovic M, Marker S, Jakobsen JC, et al. Stress ulcer prophylaxis in adult intensive care unit patients - a protocol for a systematic review. Acta Anaesthesiol Scand 2018;62:744-55.

12 Bauer M, Severus E, Möller H-J, et al. Pharmacological treatment of unipolar depressive disorders: summary of WFSBP guidelines. Int $J$ Psychiatry Clin Pract 2017;21:166-76.

13 Cleare A, Pariante CM, Young AH, et al. Evidence-Based guidelines for treating depressive disorders with antidepressants: a revision of the 2008 British association for psychopharmacology guidelines. J Psychopharmacol 2015;29:459-525.

14 Gelenberg AJ, Freeman MP, Markowitz JC, et al. Practice guideline for the treatment of patients with major depressive disorder. 3rd edn. American Psychiatric Association, 2010. 
15 Kennedy SH, Lam RW, McIntyre RS, et al. Canadian network for mood and anxiety treatments (CANMAT) 2016 clinical guidelines for the management of adults with major depressive disorder. Can J Psychiatry 2016;61:540-60.

16 Malhi GS, Bassett D, Boyce P, et al. Royal Australian and New Zealand College of Psychiatrists clinical practice guidelines for mood disorders. Aust N Z J Psychiatry 2015;49:1087-206.

17 Hirsch M, Birnbaum RJ. Switching antidepressant medications in adults, 2017. Available: http://wwwuptodatecom/index

18 Marcus SC, Olfson M. National trends in the treatment for depression from 1998 to 2007. Arch Gen Psychiatry 2010;67:1265-73.

19 National Institute for Clinical Excellence. Depression: management of depression. primary and secondary care clinical practice guideline no 23 London: NICE, 2004. Available: wwwniceorguk/pageaspx?o=235213

20 Fournier JC, DeRubeis RJ, Hollon SD, et al. Antidepressant drug effects and depression severity: a patient-level meta-analysis. JAMA 2010;303:47-53.

21 Kirsch I, Deacon BJ, Huedo-Medina TB, et al. Initial severity and antidepressant benefits: a meta-analysis of data submitted to the food and drug administration. PLoS Med 2008;5:e45.

22 Crosby RD, Kolotkin RL, Williams GR. Defining clinically meaningful change in health-related quality of life. J Clin Epidemiol 2003;56:395-407.

23 Leucht S, Fennema H, Engel R, et al. What does the HAMD mean? J Affect Disord 2013;148:243-8.

24 Moncrieff J, Kirsch I. Empirically derived criteria cast doubt on the clinical significance of antidepressant-placebo differences. Cont Clin Trials 2015;43:60-2.

25 Altman DG, Royston P. The cost of dichotomising continuous variables. BMJ 2006;332.

26 Kirsch I, Moncrieff J. Clinical trials and the response rate illusion. Contemp Clin Trials 2007;28:348-51.

27 Tennant P. Antidepressant benefits: Misinferance from ordinal scales? BMJ $2008 ; 336$.

28 Jakobsen JC, Katakam KK, Schou A, et al. Selective serotonin reuptake inhibitors versus placebo in patients with major depressive disorder. A systematic review with meta-analysis and trial sequential analysis. $B M C$ Psychiatr 2017;17:58.

29 Bagby RM, Ryder AG, Schuller DR, et al. The Hamilton depression rating scale: has the gold standard become a lead weight? Am J Psychiatry 2004;161:2163-77.

30 Ebrahim S, Bance S, Athale A, et al. Meta-Analyses with industry involvement are massively published and report no caveats for antidepressants. J Clin Epidemiol 2016;70:155-63.

31 Liberati A, Altman DG, Tetzlaff J, et al. The PRISMA statement for reporting systematic reviews and meta-analyses of studies that evaluate healthcare interventions: explanation and elaboration. BMJ 2009;339:b2700.

32 Savović J, Jones HE, Altman DG, et al. Influence of reported study design characteristics on intervention effect estimates from randomized, controlled trials. Ann Intern Med 2012;157:429-38.

33 Savović J, Turner RM, Mawdsley D, et al. Association between Risk-ofBias assessments and results of randomized trials in Cochrane reviews: the ROBES Meta-Epidemiologic study. Am J Epidemiol 2018;187:1113-22.

34 Wood L, Egger M, Gluud LL, et al. Empirical evidence of bias in treatment effect estimates in controlled trials with different interventions and outcomes: meta-epidemiological study. BMJ 2008;336:601-5.

35 Cipriani A, Furukawa TA, Salanti G, et al. Comparative efficacy and acceptability of 21 antidepressant drugs for the acute treatment of adults with major depressive disorder: a systematic review and network metaanalysis. The Lancet 2018;391:1357-66.

36 Hróbjartsson A, Emanuelsson F, Skou Thomsen AS, et al. Bias due to lack of patient blinding in clinical trials. A systematic review of trials randomizing patients to blind and nonblind sub-studies. Int J Epidemiol 2014;43:1272-83.

37 Hróbjartsson A, Thomsen ASS, Emanuelsson F, et al. Observer bias in randomised clinical trials with binary outcomes: systematic review of trials with both blinded and non-blinded outcome assessors. BMJ 2012;344:e1119.

38 Hróbjartsson A, Thomsen ASS, Emanuelsson F, et al. Observer bias in randomized clinical trials with measurement scale outcomes: a systematic review of trials with both blinded and nonblinded assessors. Can Med Assoc J 2013;185:E201-11.

39 Lundh A, Lexchin J, Mintzes B, et al. Industry sponsorship and research outcome. Cochrane Database Syst Rev 2017;2.

40 Nüesch E, Trelle S, Reichenbach S, et al. The effects of excluding patients from the analysis in randomised controlled trials: meta-epidemiological study. BMJ 2009;339.

41 Sutton AJet al. Empirical assessment of effect of publication bias on metaanalyses. BMJ 2000;320:1574-7.

42 Wisniewski SR, Rush AJ, Nierenberg AA, et al. Can phase III trial results of antidepressant medications be generalized to clinical practice? A STAR*D report. Am J Psychiatry 2009;166:599-607.

43 Kirsch I, Huedo-Medina T, Pigott H, et al. Do outcomes of clinical trials resemble those "real world" patients? A reanalysis of the STAR*D antidepressant data set. Psychology of Consciousness: Theory, Research, and Practice, 2018.

44 Gibbons RD, Hur K, Brown CH, et al. Benefits from antidepressants: synthesis of 6-week patient-level outcomes from double-blind placebocontrolled randomized trials of fluoxetine and venlafaxine. Arch Gen Psychiatry 2012;69:572-9.

45 Furukawa TA, Maruo K, Noma H, et al. Initial severity of major depression and efficacy of new generation antidepressants: individual participant data meta-analysis. Acta Psychiatr Scand 2018;137:450-8.

46 Rabinowitz J, Werbeloff N, Mandel FS, et al. Initial depression severity and response to antidepressants $v$. placebo: patient-level data analysis from 34 randomised controlled trials. Br J Psychiatry 2016;209:427-8.

47 Henssler J, Kurschus M, Franklin J, et al. Long-Term acute-phase treatment with antidepressants, 8 weeks and beyond: a systematic review and meta-analysis of randomized, placebo-controlled trials. J Clin Psychiatry 2018;79.

48 Fava GA. Can long-term treatment with antidepressant drugs worsen the course of depression? J Clin Psychiatry 2003;64:123-33.

49 Ferguson JM. Ssri antidepressant medications: adverse effects and tolerability. Prim Care Companion J Clin Psychiatry 2001;3:22-7.

50 Farnsworth KD, Dinsmore WW. Persistent sexual dysfunction in genitourinary medicine clinic attendees induced by selective serotonin reuptake inhibitors. Int J STD AIDS 2009;20:68-9.

51 Reefhuis J, Devine 0, Friedman JM, et al. Specific SSRIs and birth defects: Bayesian analysis to interpret new data in the context of previous reports. BMJ 2015;351.

52 Fava GA, Gatti A, Belaise C, et al. Withdrawal symptoms after selective serotonin reuptake inhibitor discontinuation: a systematic review. Psychother Psychosom 2015;84:72-81.

53 Davies J, Read J. A systematic review into the incidence severity and duration of antidepressant withdrawal effects: are guidelines evidencebased? Addictive Behaviors 2018.

54 Geddes JR, Carney SM, Davies C, et al. Relapse prevention with antidepressant drug treatment in depressive disorders: a systematic review. Lancet 2003;361:653-61.

55 Reid S, Barbui C. Long term treatment of depression with selective serotonin reuptake inhibitors and newer antidepressants. BMJ 2010;340:c1468.

56 National Institute for Health and Care Excellence (NICE). Depression in adults: recognition and management. Clinical guideline [CG90] Published date: October 2009, 2016.

57 Parikh SV, Quilty LC, Ravitz P, et al. Canadian network for mood and anxiety treatments (CANMAT) 2016 clinical guidelines for the management of adults with major depressive disorder. Can J Psychiatry 2016;61:524-39.

58 Karyotaki E, Smit Y, Holdt Henningsen K, et al. Combining pharmacotherapy and psychotherapy or monotherapy for major depression? A meta-analysis on the long-term effects. J Affect Disord 2016;194:144-52.

59 Khan A, Faucett J, Lichtenberg P, et al. A systematic review of comparative efficacy of treatments and controls for depression. PLoS One 2012; 7:e41778.

$60 \mathrm{Krogh}$ J, Hjorthøj C, Speyer H, et al. Exercise for patients with major depression: a systematic review with meta-analysis and trial sequential analysis. BMJ Open 2017;7:e14820. 
61 Crowe L, Butterworth P. The role of financial hardship, mastery and social support in the association between employment status and depression: results from an Australian longitudinal cohort study. BMJ Open 2016;6:e009834.

62 Lorant V, Croux C, Weich S, et al. Depression and socio-economic risk factors: 7-year longitudinal population study. Br J Psychiatry 2007;190:293-8.
63 Eiring 0, Landmark BF, Aas E, et al. What matters to patients? A systematic review of preferences for medication-associated outcomes in mental disorders. BMJ Open 2015;5:e007848.

64 Rosenblat JD, Simon GE, Sachs GS, et al. Factors that impact treatment decisions: results from an online survey of individuals with bipolar and unipolar depression. Prim Care Companion CNS Disord 2018;20. doi: $10.4088 /$ PCC. $18 \mathrm{~m} 02340$ 